\title{
A Distributed Approach based on Transition Graph for Resolving Multimodal Urban Transportation Problem
}

\author{
Mohamed El Moufid ${ }^{1}$, Younes Nadir ${ }^{2}$, Khalid Boukhdir ${ }^{3}$, Siham Benhadou ${ }^{4}$, Hicham Medromi ${ }^{5}$ \\ LRI, National High School of Electricity and Mechanics, Casablanca, Morocco \\ Foundation of Research, Development and Innovation in Sciences and Engineering, Casablanca, Morocco
}

\begin{abstract}
All over the world, many research studies focus on developing and enhancing real-time communications between various transport stakeholders in urban environments. Such motivation can be justified by the growing importance of pollution caused by the transport sector in urban areas. In this work, we propose an approach of assistance for displacement in urban environment taking advantages of multimodal urban transportation means, where several modes of public transports are available. In addition, we also consider the possibility of using both private modes of transport and cities parking. The proposed distributed approach described in this paper is based on an abstraction of a city multimodal graph according to the available modes of public transport and road traffic and transition graph approach to move from a mode to the other mode. Numerical results are developed to justify the effectiveness of our approach.
\end{abstract}

Keywords-Multimodal transport; distributed approach; transition graph

\section{INTRODUCTION}

Displacements in urban environments are one of the major problems facing most supercities around the world. In addition to the pollution and time waste generated by traffic jams expectations while looking-up for parking spots to park private vehicles [1], urban mobility favors certain health disturbances among cities citizens. In recent year authorities have encouraged the use of a diversity of transport modes in order to reduce the impact of this problem [2].

Several solutions have then been developed to help users to choose the optimal path to follow to reach their destinations. Early solutions concerned each a particular mode of public transport and each operator proposed a system that manages its own network. Subsequently, other solutions made it possible to propose the best optimal paths by combining two or more modes of public transports [3] [4].

Currently, researches are moving towards the proposal of an urban mobility management system by considering both all available cities public modes of transports, traffic road states and the availability of city car parks. Thus, the optimal route for a user would be to drive his private vehicle to a parking lot, then take a series of public transport lines.

In order to be able to ally with the development of the city's urban transport infrastructure, urban travel assistance services must be able to consider the growing complexity of public transport networks, road traffic density and daily requests for parking space.

Several studies examine the possibility of establishing both multimodal transport networks models, road states and the availability of car parks in order to propose approaches that calculate and determine the most appropriate paths for a user's request. These approaches must be robust enough to keep their efficiency and effectiveness as the complexity of the multimodal network and the amount of data to be processed increases.

The major issues in the development of these approaches can then be considered in two aspects:

1) Management of heterogeneous data: Different modes correspond to a set of navigation datasets which are acquired, stored and managed by different public or private organizations. Likewise, car parks management systems make it possible to retrieve data concerning the availability of car parks at a given moment and to predict the occupancy rate for a later date. By the same token, road traffic supervision systems provide real time data not only from traffic roads states but also a full history of occupancy rate related to road traffic arcs [5].

2) Multimodal network and optimal path finding approach: Different types of data should be considered in determining the optimal path in a multimodal network. Various works then seek to elaborate the problem modeling approach which allows an adequate structuration and an easy accessibility to the information which is necessary for different computations [6]. Likewise, the resolution approach must also be sufficiently powerful and flexible to be able to maintain its performance when increasing the size of the multimodal network [7].

In the present paper we are going to present a new model of the multimodal network based on the graph theory and a parallel distributed approach to propose optimal paths between two points in a city.

The rest of the paper will be developed as follows: in Section II we present definitions of the concepts necessary for the description of the problem and the proposed solution. These definitions concern the modeling of the multimodal transport network using graph theory. Then we proceed in the third part to the mathematical modeling of the problems in 
order to propose the approaches of resolution allowing at first to reduce the complexity of the problems and then to propose the optimal solutions. We conclude our work with a conclusion and perspectives.

\section{PROBLEM FORMULATION}

In this section, we present some necessary definitions for the formulation of the optimal path problem in a multimodal transport network using graph theory approach. The particularity of the problem is that the multimodal transport network contains several subnetworks, each subnetwork refers to either a particular mode of transport or the road traffic network of the city.

The model should propose an optimal structuring of the data in such a way that the system could have rapid access to each arc at any subnetwork of the multimodal network. Then it would be able to react in real time on following any change that may happen on an arc of the multimodal network. Different models have been proposed to consider the dynamic particularity mentioned above, generally based on graph theory: hypergraph theory [8] [9] [10], hierarchical graph [11], colored graph approach [12] and transfer graph approach [13]. However, most models fail to consider the following three components of a city's multimodal network simultaneously: public transport modes, road traffic network and city car parks [14].

In our study, we consider a global multimodal network composed of: a set of monomodal public transport networks, a set of parking spaces and a traffic road network. Each component of the multimodal network has its own specific parameters.

So, in regard to the resolution approach, the procedure of calculating the shortest path should be able to keep its efficiency and computing power when changing the size of the multimodal network; In most studies, the optimal path resolution approach in a multimodal network is considered as a whole. Thus, to perform calculations, the approach must traverse all nodes and arcs of the said network, which slows down the computation process and reduces the system performances.

Wherefore, in the approach, the calculations are done in a parallel and distributed way for each component of the global network. Thus, to calculate the optimal path between two points of a multimodal network, our approach consists on making intermediate calculations for each sub graph before considering the whole multimodal network. This need to reduce the size of the graph is justified by the complexity of the problem. Thus, with a graph of $n$ nodes, $e$ edges and $m$ modes of transport, the corresponding graph generated will have a complexity in at least of $\mathcal{O}\left(n e^{3}\right)$ [15] [16].

In the following, we define some concepts that will allow to present our model and our approach of resolution:

Let $G=(N, E, M, P)$ denotes a multimodal transport graph, where $N_{j}=\left\{n_{j 1}, \ldots, n_{j l}\right\}$ is a set of nodes belonging to the mode $j, \quad \mathrm{M}=\left\{m_{1}, \ldots, m_{k}\right\}$ is a set of modes, $P=$ $\left\{p_{1}, \ldots, p_{s}\right\}$ is a set of packing spaces.
An edge defined by $e_{i}=\left(n_{j q}, n_{j r}\right)_{m_{j}}$ expresses that it is possible to go from node $n_{\mathrm{jq}}$ to node $\mathrm{n}_{\mathrm{jr}}$ using transport mode $\mathrm{m}_{\mathrm{j}}$. A value $f^{r}{ }_{e_{i}}\left(t_{k}\right)$ is associated to each edge $e_{i}$ indicating the cost of the edge $e_{i}$ at departure time $t_{k}$ according to the criterion $r$ (e.g., distance or duration).

\section{A. Définition 1: Multimodal Path}

Given a multimodal transport graph $G=(N, E, M, P)$. A multimodal path $p_{n_{i o}, n_{j d}}$ is a sequence of possible edges to go from the node $n_{i o}$ to the node $n_{j d}$, $p_{n_{i o}, n_{j d}}=\left(\left(n_{i}, n_{2}\right)_{m_{o}},\left(n_{2}, n_{3}\right)_{m_{p}}, \ldots,\left(n_{j-1}, n_{j}\right)_{m_{d}}\right)$.

\section{B. Définition2: Cost Function}

The vector-valued function: $f_{r}\left(p, t_{0},\right): \quad P * T \rightarrow R$ represents the cost of the path $p$ departing at time $t_{0}$ according to the criteria $r . R$ is a set of vectors, where each vector represents a criterion.

\section{Definition 3: Optimal Path Problem}

Given a graph $G=(N, E, M, P)$, the optimal path problem according to the criteria $r$ consists in calculating a path $p_{n_{i o}, n_{j d}}$ from node $o$ to node $d$ departing at $t_{0}$ where $f_{r}\left(p, t_{0}\right)$ is minimal. This is called the optimal path (OP).

\section{Transition Graph}

Given a multimodal graph $G=(N, E, M, P)$, the transition graph is defined as $T_{g}=\left(C, T_{r}\right)$ where $C=\left\{C_{1}, C_{2}, \ldots, C_{k}\right\}$ is the set of monomodal graphs, and $T_{r}$ is the set of virtual transition edges which interconnect them. Each component $C_{i}=\left(N_{i}, E_{i}, M_{i}, P_{i}\right)$ is such that $N=\bigcup_{i \in\{1, \ldots, k\}} N_{i}, E=$ $\bigcup_{i \in\{1, \ldots, k\}} E_{i}, P=\bigcup_{i \in\{1, \ldots, k\}} P_{i}$.

The transition graph model consists in abstracting different modes of transport on the same map; we can distinguish in a transfer graph $T_{g}$ between two groups of paths: intracomponents paths and inter-components paths. An intercomponent path refers to a path that connects two nodes belonging to distinct modes of transport [17], while an intracomponent path defines a path that links two nodes belonging to the same mode of transport

Figure 1 illustrates an example of a transfer graph, where Mode 1, Mode 2 and Mode 3 represent three modes of public transport and Road Graph represent the road network. distinguishes between two categories of monomodal graphs can be described as follow:

- Mode Graph represent public modes (Mode 1, Mode 2, Mode 3): nodes represent stations of the mode and edges represent the paths of the transport mode (Tramway, bus, subway ...). Parameters of the graphs (departure times at a node, estimated duration to travel an arc ...) depend on the mode of transport and are given by the public transport operator. These parameters can be updated by a user or an operator once a disturbance occurs on a line of the mode.

- Road Graph represents the network of the road traffic: local nodes represent the intersections of the paths and transfer nodes represent car parks. 


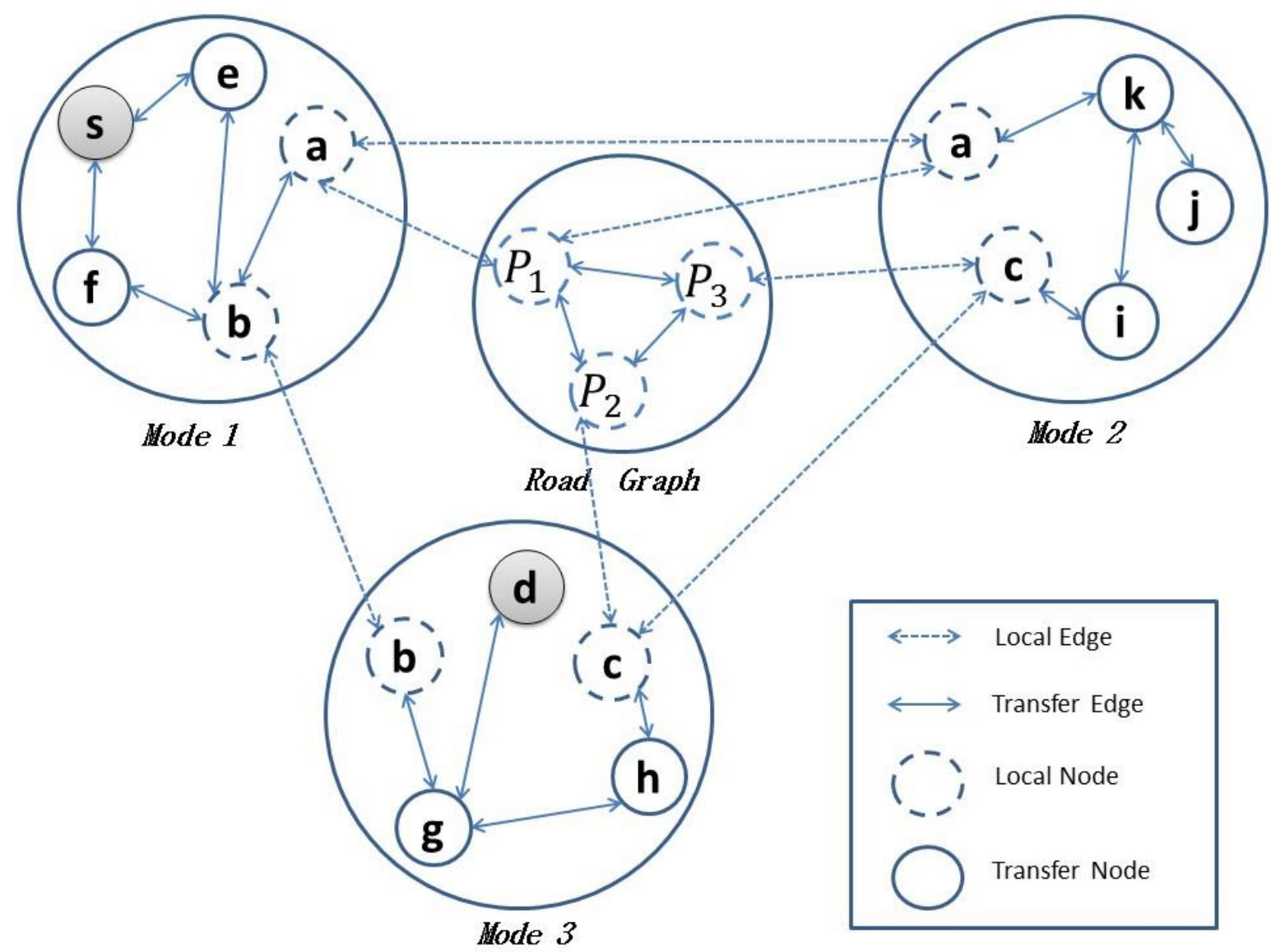

Fig. 1. Transition Graph Illustrative Case.

A citizen can drive his own car from an initial point to a second point, then park his car in a parking lot and finish his travel with a public mode of transport. Different parameters related to the state of an arc of a road network are updated by users who browse it in real time.

In the current study, transfer node represents walking movements either to go from a parking space to a public transport mode (if the latest mode of transport is a private vehicle), or to go from a station of a mode of transport to a station of another mode of transport.

\section{Problem Resolution and Results}

The objective function is a multi-objective function. Indeed, by varying the multimodal path between two nodes of the multimodal graph, various parameters can vary (duration, cost, level of comfort ...).

Let $p_{n_{i o}, n_{j d}}$ be a multimodal path from node $n_{i o}$ to node $n_{j d}$. The optimal path between a node $i$ belonging to a mode $m$ to a node $j$ belonging to a mode $n$ according to the criteria $k$ can be defined as follow:

$F_{k}=\min \sum_{i, j, m, n} x_{i_{m} j_{n}} * f_{k}^{t_{0}}\left(x_{i_{m} j_{n}}\right)$ $x_{i_{m} j_{n}}:$ logical variable defines whether the $e_{i_{m} j_{n}}$ edge linking a node $i$ belonging to a mode $m$ to a node $\mathrm{j}$ belonging to a mode $\mathrm{n}$ is used or not.

$f_{k}^{t_{0}}\left(x_{i_{m} j_{n}}\right)$ : cost function of the direct arc linking a node $i$ belonging to a mode $m$ to a node $\mathrm{j}$ belonging to a mode $n$ departing at time $t_{0}$.

The optimal global function should consider all possible criterions and proposes the set of optimal paths according to all criterions.

In our study, for problem simplification reasons, we consider that the optimal global function is a direct weighting of optimal paths according to each criterion [18]. follow:

The minimization of the global function can be written as $F=\min \sum_{k} w_{k} * F_{k}$

Where $w_{k}$ are weighting coefficients and refers to a user's preference according to the criteria $k$.

The optimization of the global function is subject to the following constraints:

Where: 


$$
\begin{aligned}
& \sum_{j_{n}} x_{i_{m} j_{n}}-\sum_{i_{m}} x_{i_{m} j_{n}} \quad= \\
& \left\{\begin{array}{cc}
-1 & \text { if } \quad \forall=o \\
0 \quad & \forall j \in V, j \neq o, d \\
1 \quad \text { if } \quad i=d
\end{array}\right. \\
& \sum_{m, n} x_{i_{m} j_{n}} \leq 1, \quad \forall(i, j) \in E, \quad(m, n) \in M \\
& \sum_{l} x_{l_{v} i_{v}} \geq x_{i_{v} j_{v}} \quad \forall\left(i_{v}, j_{v}\right) \in M_{v}
\end{aligned}
$$

Where $v$ refers to a private mode.

The equation (3) ensures that each node of the proposed paths is visited once at maximum.

The constraint (4) ensures that each arc is traveled by one mode of transport at maximum and the constraints (5) ensures that if a user leaves his car in a city's parking lot, the rest of the proposed path must not propose a return to his private vehicle.

The problem of the optimal path according to $r$ criterions in a multimodal transport network composed of $n$ nodes and $e$ is a multiobjective function with complexity of $\mathcal{O}\left(\mathrm{rn} e^{3}\right)$ [19]. In the literature, the common difficulty resides in maintaining performance of the approach when the considered network becomes larger and more complex [20] [21].

In the proposed work, we propose an approach allowing to reduce the complexity of the problem based particularly on The Depths-First-Search algorithm described in Algorithm 2 which is at a difficulty of $\mathcal{O}(|V|+|E|)$. The approach is based on the following ideas:

1) Reduce the size of the whole multimodal graph and assign for each mode of public transport/ road traffic network the corresponding sub-graph.

2) Look for the possible paths between the nodes and then evaluate them according to each desired criterion (duration, cost...), which makes it possible to go from a complexity of $\mathcal{O}\left(\right.$ rne $\left.^{3}\right)$ to a $\mathcal{O}(|V|+|E|)$ problem complexity.

3) Evaluate the possible paths according to each classified parameters of the user then assign weighting coefficients. this is the fastest approach for multi-objective evaluation [16].

4) In order not to repeat similar calculations we propose at first to check if the system has already responded to a similar request. If the case, it exploits them. Otherwise, it proceeds normally to the calculations.

In order to validate the performance of our proposed approach, and evaluate the win of each of the proposed ideas, we performed a number of experimental tests. We test and compare our approach in terms of execution time according to the size of the network considered.

We consider 10 main networks varying from 50 nodes to 10000 nodes. We consider three main approaches:

1) The whole graph is considered, and the Algorithm 1 is considered from the step 3 .

2) The transition graph is considered, but the second step is skipped in order to do all necessary calculations.
3) The whole algorithm is considered and previous requests and proposed multimodal paths are taken into consideration in order to reduce calculations.

Thus, following a user's request, an optimal path should be calculated as described in Algorithm 1.

Algorithm 1: Resolution Approach Algorithm

Require: (Multimodal-Graph, Start-Node, Destination-Node, Levels of preferences)

1. Create the simplified transition associated graph based on the multimodal-Graph.

2. Search in the Database for similar queries.

3. Determine possible multimodal paths from the starting point to the desired destination.

The used method for in this step is defined in Algorithm 2.

4. Evaluate possible multimodal paths according to the levels of preferences defined by the user.

5. Classify the set of evaluated paths according to user preferences.

6. Propose the optimal paths to user request.

7. Validate the chosen path and save the experience.

The second step of the Algorithm 1 is based on the DFS approach defined in Algorithm 2

Algorithm 2:Adapted DFS Algorithm

Require: (Transition-Graph, Start-Node, Destination-Node)

Put anyone of the graph's vertices on top of a stack.

\section{Repeat \{}

While stack is not empty \{

Take the top item of the stack and add it to the visited list.

Create a list of that vertex's adjacent nodes. Add the ones which aren't in the visited list to the top of stack.\}

Until stack is empty\}

Results of simulations of the programs under MATLAB student-use software using a configuration computer: Intel Core i3-370M, 2.40GHz and RAM 4 GB, show at Figure 2 that from a network of 1000 nodes, the last two approaches show a high efficiency compared to the first approach. Indeed, in the second approach, the global graph is abstracted into subgraphs, this distributed approach makes it then possible to simplify the problem and consequently to reduce the execution time. 


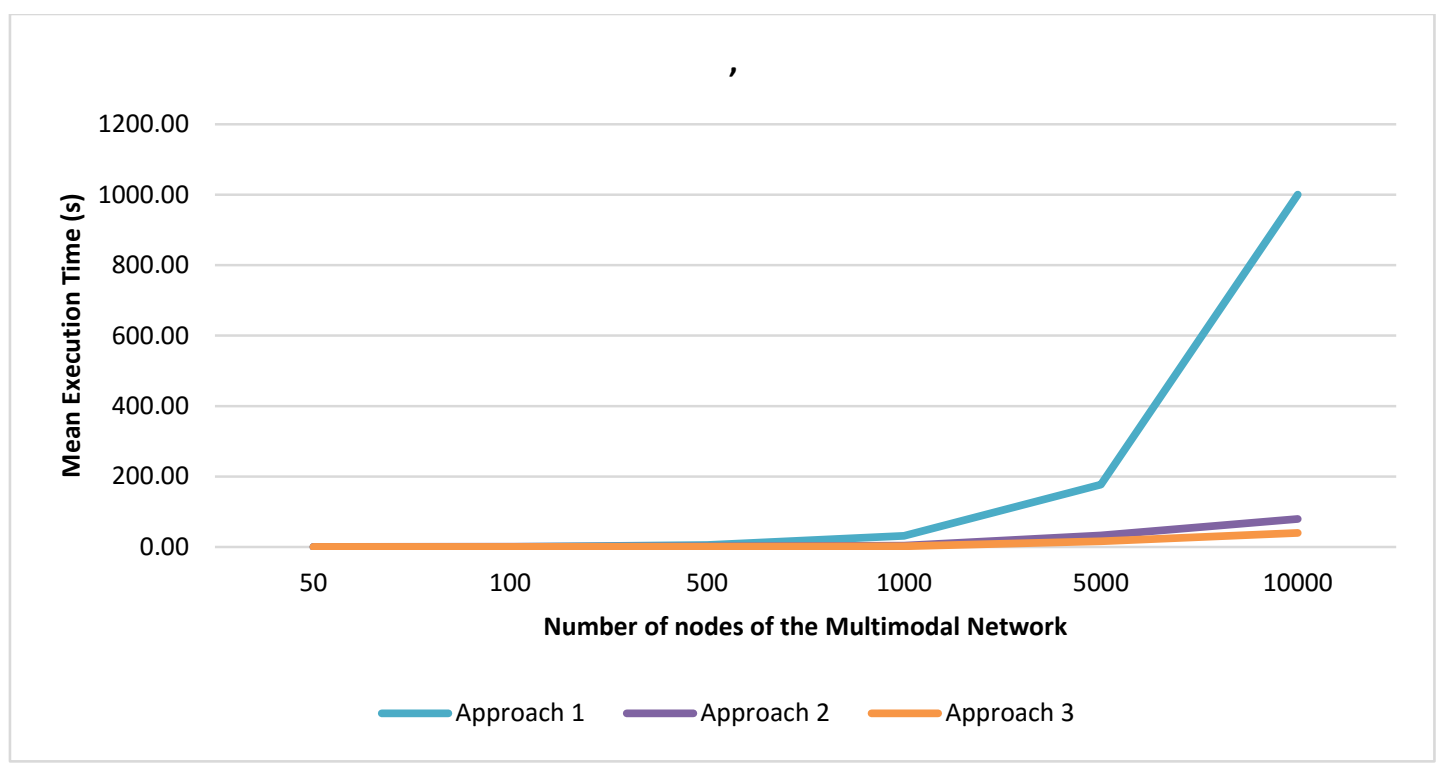

Fig. 2. Results of Comparisons.

The third approach, in addition to the advantages of the distributed aspect of its execution, it makes it possible to benefit from the previous experiences recorded in the database of the system. It consequently eliminates some calculation operations and subsequently reduces the execution time.

\section{CONCLUSION AND PERSPECTIVES}

The proposed work is useful to find the optimal multimodal paths in a multimodal network of transportation composed of a set of monomodal public transportation networks (Tramway, train, metro...), of road traffic network and a set of available parking in a city.

The global multimodal network is abstracted into subgraphs where each graph refers either to a monomodal public transportation network or a traffic road network. The distribution of the problem makes it easier to solve, and allows acting on a sub-graph without affecting other monomodal subgraphs.

The proposed resolution approach takes advantage of the simplification of the problem obtained by its abstraction and shows good results compared to the case where the whole network is considered in calculations.

In the third approach, artificial intelligence is integrated to identify whether the system has already responded to a similar request. In this, calculations aren't to be redone which impact positively the execution time and improve the efficiency of the system.

As perspective, different possible approaches for artificial intelligence could be compared in order to identify similarities and choose the most appropriate for the problem.

\section{REFERENCES}

[1] T. Yogesh and P. Mr.M.D, "Advance Prediction of Parking Space Availability and other facilities for Car parks in Smart Cities," International Research Journal of Engineering and Technology (IRJET), vol. 3, no. 5, pp. 2225-2228, 2016.

[2] W. R.C.P, S. W.Y., Y. Linchuan, L. Y.C. and W. S.C., "Public transport policy measures for improving elderly mobility," Transport Policy, vol. 63, pp. 73-79, 2018.

[3] L. David and L. Angélica, "Techniques in Multimodal Shortest Path in Public Transport Systems," Transportation Research Procedia, vol. 3, pp. 886-894, 2014.

[4] C. Lin, C. Peng and P. Jianping, "Delay Minimization for Data Dissemination in Large-Scale VANETs with Buses and Taxis," IEEE Transactions on Mobile Computing, vol. 15, no. 8, pp. 1939 - 1950, 2016.

[5] E. F. Nour-Eddin, L. Henry and k. Ajeesh, "Data fusion in intelligent transportation systems: Progress and challenges - A survey," Information Fusion, vol. 12, no. 1, pp. 4-10, 2011.

[6] L. Chao-Lin, P. Tun-Wen, C. Chun-Tien and H. Chang-Ming, "Pathplanning algorithms for public transportation systems," in IEEE Intelligent Transportation Systems. P, Oakland, CA, 2001.

[7] L. Liu, "Data Model and Algorithms for Multimodal Route Planning," München, 2010.

[8] S. Pallottino and S. Nguyen, "Equilibrium traffic assignment for large scale transit networks," European Journal of Operational Research, vol. 37, no. 2, pp. 176-186, 1988.

[9] A. D. Febbraro and S. Sacone, "An online information system to balance traffic flows in urban areas," in Proceedings of the 36th IEEE Conference on Decision and Control, San Diego, CA, 1997.

[10] A. Lozano and G. Storchi, "Shortest viable path algorithm in multimodal networks," Transportation Research Part A: Policy and Practice, Elsevier, vol. 35, no. 3, pp. 225-241, 2001.

[11] M. Bielli, A. Boulmakoul and H. Mouncif, "Object modeling and path computation for multimodal travel systems," European Journal of Operational Research, vol. 175, no. 3, pp. 1705-1730, 2006.

[12] A. Ensor and F. Lillo, "Colored-Edge Graph Approach for the Modeling of Multimodal Transportation Systems," Asia-Pacific Journal of Operational Research, vol. 33, no. 1, pp. 1-21, 2016. 
[13] H. Ayed, C. Galvez-Fernandez, Z. Habbas and D. Khadraoui, "Solving time-dependent multimodal transport problems using a transfer graph model," Computers \& Industrial Engineering, vol. 61, no. 2, pp. 391-401, 2011.

[14] D. M. Sergio and R. Silvia, "An architecture for a Mobility Recommander System," Procedia Computer Sscience, vol. 98, pp. 425430, 2016.

[15] Z. Athanasios and W. Whitney, "An intermodal optimum path algorithm for multimodal networks with dynamic arc travel times and switching delays," European Journal of Operational Research, vol. 125, no. 3, pp. 486-502, 2000.

[16] T. Gräbener, "Calcul d'itinéraire multimodal et multiobjectif en milieu urbain: Modélisation et simulation," Université des Sciences Sociales, Toulouse I, France, 2010.
[17] Z. Jianwei, L. Feixiong, A. Theo and T. Harry, "A multimodal transport network model for advanced traveler information systems," in Procedia Social and Behavioral Sciences, 2011.

[18] D. Kalyanmoy, "Multi-objective Optimization," in Burke E., Kendall G. (eds) Search Methodologies. , Boston, Springer, 2013, pp. 403-449.

[19] F. Reza Zanjirani, M. Elnaz, S. W.Y and . R. Hannaneh, "A review of urban transportation network design problems," European Journal of Operational Research, vol. 229, no. 1, pp. 281-302, 2013.

[20] S. Juliana Verga , Y. Akebo, C. S. Ricardo and S. Wesley Vagner Inês, "Urban Transport and Traffic Systems: An Approach to the Shortest Path Problem and Network Flow Through Colored Graphs," in Nazário Coelho V., Machado Coelho I., A.Oliveira T., Ochi L. (eds) Smart and Digital Cities. Urban Computing. Springer,, Cham, 2019.

[21] C. Xinyuan and K. Inhi, "Modelling Rail-Based Park and Ride with Environmental Constraints in a Multimodal Transport Network," Journal of Advanced Transportation, vol. 2018, pp. 15 pages,, 2018. 